\title{
Report from the 24th Congress of the European Association of Urology, Stockholm, Sweden, 17-21 March 2009, dedicated to renal carcinoma
}

\author{
Chiara Paglino
}

Published online: 16 June 2009

(c) Springer-Verlag 2009

Once again, this year's annual congress of the European Association of Urology (EAU) proved to be of great interest, not only for those more directly involved, the urologists, but also for who, like me, participated as an observer from the field of medical oncology.

In fact, as usual, genitourinary oncology made up an important part of the congress events. Although surgical aspects obviously took up much attention, there were less numerous and stimulating presentations. These concerned, in particular, not only experimental oncology but also nonsurgical treatment of urological neoplasias.

There were 1,033 abstracts together with over 40 video presentations. These have been published in the traditional supplement of the Platinum Journal (the by now renowned and influential European Journal of Urology). Of these, 136 were about renal neoplasias.

As could be expected, just under half of these abstracts concerned surgery. However, there were many with a wider appeal related to basic research (22), to diagnosis (34) and to non-surgical treatment (22) of renal neoplasias.

Among the extremely high-quality papers presented concerning experimental issues, those which, in my opinion, proved to be the most intriguing were the identification of a possible new tumor suppressor gene presented by a Chinese group [1], and the description of two different angiogenic phenotypes predictive of response to antiangiogenic therapies presented by a French group [2].

In the first abstract, Zhang and colleagues [1] evaluated by means of semi-quantitative RT-PCR the expression of a

C. Paglino $(\square)$

Medical Oncology,

IRCCS San Matteo University Hospital Foundation,

Piazzale C. Golgi 19, 27100 Pavia, Italy

e-mail: chiara_paglino@fastwebnet.it recently recognized oncosuppressor gene, $K S 1$ located on chromosome 16 at $16 q 23$, on the 6 renal carcinoma cell lines and 81 neoplastic and normal tissue samples. The state of methylation of the KSI promoter gene was, therefore, evaluated and the effect of the re-expression of the gene itself on the clonogenicity of the tumor cells was examined.

The authors showed a downregulation and a methylation of the KS1 gene in all the cell lines; more than $42 \%$ in the tumor samples versus only $5.7 \%$ in the normal tissue samples.

Also, the state of methylation of the KS1 gene correlated with the TNM staging and with the degree of nuclear differentiation. Finally, the re-expression of the KS1 gene in 'silenced' cells resulted in a significant inhibition in growth of tumor cells and of their clonogenicity.

In conclusion, $K S 1$ appeared to be a potentially important oncosuppressor gene in renal carcinoma. Its methylation state could be used as a biomarker for these neoplasias with clear and important implications for diagnosis and prognosis.

In the second abstract [2], Bigot and colleagues analyzed 75 renal carcinoma samples from patients undergoing radical nephrectomy. Immunohistochemical analysis of the differences in intratumoral angiogenic 'architecture' classified these as: mature (type 1) or immature (type 2) and correlated them with the prognosis of their patients. They then analyzed the alterations in the VHL gene (mutations, deletions and hypermethylation of the promoter) in these neoplasias and correlated them with the angiogenic phenotype identified by the immunohistochemical analysis.

The authors observed a correlation both between mature angiogenic phenotype and a better prognosis, and between immature phenotype and a worse prognosis. They also 
demonstrated how the different phenotype is strictly dependent on the status of the $V H L$ gene.

Among the abstracts dedicated to diagnosis, I think it is worth drawing attention to that of an Austrian group [3] who demonstrated the sensitivity and specificity of dynamic contrast-enhanced MRI in distinguishing the nature of small renal masses.

Among the remaining abstracts, certainly of greater oncological interest, two are worthy of note: that of Staehler and colleagues [4], and that reporting the experience of a number of Italian centers in the treatment of patients with advanced renal carcinoma with the two possible sequences of Sorafenib and Sunitinib [5].

In the first abstract, the German group suggests that the association of Sorafenib or Sunitinib with radiotherapy can make this latter more effective, and can even re-sensitize to radiotherapy already treated and progressive lesions [4]. This observation seems to go against one of the cornerstones of radiation biology being that radiotherapy works better in oxygenated tissue and that the reduction of the tumoral vascularization by antiangiogenic drugs, reducing the supply of oxygen to the tumor, could be counterproductive. This abstract could be strongly criticized for its retrospective approach. However, the experience it reports is even more interesting in the light of at least one important experimental study which instead suggests the usefulness of combining an antiangiogenic, Sorafenib, with radiation. This approach takes advantage of the resultant unexpected synergy which seems to depend both on the treatment schedule and on the cell cycle state of the neoplasias treated [6].

The Italian experience analyzed the overall progression free survival (PFS) of 119 patients treated with Sorafenib and then Sunitinib or viceversa. (This study was later updated and the poster presentation in Stockholm included 170 patients, the largest study cohort on this subject to date.) Although, given its retrospective nature, the data resulting from this study should be interpreted with caution, the message which emerges, and which is confirmed in all similar but less numerous retrospective analyses available, is that there is no cross-resistance between the two drugs.

The second point which emerges from this analysis and which, once again, comes up repeatedly in all the analyses of sequential therapy known so far, is that the SorafenibSunitinib sequence seems able to induce a better overall PFS (17.2 months) with respect to the inverted sequence (11.7 months for the Sunitinib-Sorafenib sequence). This is certainly intriguing but means that even greater caution (if that is possible) is necessary and prospective studies aimed at confirming or disproving this are certainly merited and of interest.

In conclusion, I have one suggestion to make to those oncologists who are involved in genitourinary oncology and renal carcinoma in particular. In the midst of so many (and quite honestly, too many!) congresses which we attend, let us take the EAU into careful consideration. This one is really worth it!

\section{References}

1. Zhang Q, Ying JM, Poon FF et al (2009) Aberrant methylation of KS1 - a candidate tumor suppressor in renal cell carcinoma and its relationship to clinicopathological features. Eur J Urol 8(Suppl 4):297 (abs. 705)

2. Bigot P, Edeline J, Vigneau C et al (2009) The prognostic importance of angiogenic phenotype in renal cell carcinoma. Eur J Urol 8(Suppl 4):298 (abs. 711)

3. Abd Ellah MMH, Pallwein LP, Kremser C et al (2009) Role of dynamic contrast-enhanced MRI in the assessment of small renal masses. Eur J Urol 8(Suppl. 4):154 (abs. 133)

4. Staehler MD, Haseke N, Zilinberg E et al (2009) Effectivity of radiation therapy in combination with multi-kinase inhibition based on Sorafenib or Sunitinib in progressive metastatic renal cancer. Eur J Urol 8(Suppl 4):156 (abs. 143)

5. Porta C, Procopio G, Sabbatini G et al (2009) Retrospective analysis of the sequential use of Sorafenib and Sunitinib in patients with advanced renal cell carcinoma (RCC). Eur J Urol 8(Suppl 4):183 (abs. 252)

6. Plastaras JP, Kim SH, Liu YY et al (2007) Cell cycle-dependent and schedule-dependent antitumor effects of Sorafenib combined with radiation. Cancer Res 67:9443-9454 